\title{
CARACTERIZAÇÃO DAS COOPERAÇÕES INTERINSTITUCIONAIS NO ESTADO DO PARANÁ: UMA ANÁLISE SOB A ÓTICA DAS UNIVERSIDADES
}

\author{
CHARACTERIZATION OF INTERINSTITUTIONAL \\ COOPERATION IN PARANÁ STATE: \\ AN ANALYSIS FROM THE UNIVERSITIES PERSPECTIVE
}

Recebido em: 07/01/2016 A Aprovado em: 18/03/2016
Avaliado pelo sistema double blind review
Editora Científica: Claudia Stadtlober
DOI 10.13058/raep.2016.v17n2.370

\section{FABRICIO BARONMUSSIfabricio_mussi@hotmail.com PONTIFÍCIA UNIVERSIDADE CATÓLICA DO PARANÁ}

\begin{abstract}
RESUMO
A presente pesquisa buscou caracterizar as cooperações em que a universidade se constitui em uma das partes envolvidas e, em especial, os relacionamentos nos moldes Universidade-Empresa (U-E), Universidade-Universidade (U-U) e Universidade-Institutos de Pesquisa (U-IP). Na pesquisa, de natureza descritiva, foram empregados métodos de natureza qualitativa e quantitativa. Constatou-se que parte significativa do referencial disponível a respeito desse tema ainda está centrada nas relações entre universidades e empresas. Para os itens que compõem as dimensões de facilitadores, dificultadores, motivadores e determinantes da cooperação interinstitucional, verificouse que existem diferenças de acordo com o tipo de parceiro com o qual as instituições acadêmicas se envolvem. Observou-se, ainda, que cada tipo de interação possui determinadas especificidades com relação ao instrumento contratual que norteia a cooperação. Outra constatação concerne à predominância de pesquisas conjuntas na área de ciências exatas. Uma vez identificados os fatores que interferem nas cooperações interinstitucionais, sugere-se, como potencial pesquisa futura, avaliar a contribuição de cada fator por intermédio das técnicas de regressão quantílica.
\end{abstract}

Palavras-chave: Cooperação interinstitucional; Universidades; Empresas; Institutos de pesquisa.

\section{ABSTRACT}

This study aimed to characterize the collaboration in which the university is one of the parties involved, in particular the model relationships between University Enterprise (U-E), University-University (U-U), and University-Research Institutes (U-RI). In this descriptive research, the methods used were of qualitative and quantitative nature. It was found that a significant part of the reference available regarding this topic is still centered in the relationship between universities and companies. For the items that compose the dimensions of facilitators, barriers, motivators, and determinants for the interinstitutional cooperation, it was found that there were differences according to the type of partner which the academic institutions are involved with. In addition, it was also observed that each type of interaction has certain specificities regarding the contractual instrument which guides the cooperation. Another finding concerns the prevalence of the joint research in the field of the exact sciences. Once identified the factors that interfere with the interinstitutional cooperation, it is suggested as a potential future research the assessment of the contribution of each factor through the quantile regression techniques.

Keywords: Interinstitutional Cooperation; Universities; Enterprise; Research Institutes. 


\section{INTRODUÇÃO}

Diversos estudos têm mostrado a relevância das relações entre os agentes que, segundo a literatura, compõem um sistema nacional de inovação e, mais especificamente, entre universidades, institutos de pesquisa e empresas. Assim, a exploração do tema alcança consenso em determinadas frentes - como as atribuições do governo nos sistemas nacionais de inovação (ETZKOWITZ; LEYDESDORFF, 1998, 2000; ETZKOWITZ, 2002; ETZKOWITZ; MELLO, 2004); a relevância da gestão dos relacionamentos entre empresas e universidades (ANDREASSI; VANCONCELLOS; SIMANTOB, 2002; PORTO, 2002; PORTO; PLONSKI, 2000). Já em outras frentes, ainda, encontram-se abertas algumas lacunas para a pesquisa - como a cooperação entre pesquisadores nos diversos campos da administração (ROSSONI; GUARIDO-FILHO, 2007), a cooperação nas universidades (LYLES, 1999) e a avaliação das atividades acadêmicas, no que se refere a pesquisas, publicações e citações (HUANG; CHANG; CHEN, 2006).

As pesquisas sobre os meios aplicados para acelerar e consolidar a interação de universidades, institutos de pesquisa e empresas têm variado quanto à natureza. Algumas dessas investigações são desenvolvidas com o propósito de criar relatórios ou com o objetivo de realizar auditorias sobre a forma e a frequência da interação. Castells (1999) e Schibanny e Polt (200I) salientam que o ritmo com que surgem alianças entre instituições e entre pesquisadores com o propósito de realizar trabalhos conjuntos de pesquisa está relacionado à cadência com que algumas tecnologias, em especial as tecnologias da informação, desenvolvem-se. Outras fontes de estímulos podem ser representadas pelas crescentes e contínuas empreitadas de órgãos de fomento nas esferas federal e estadual (principalmente, por intermédio das Fundações de Amparo à Pesquisa), assim, promovendo as associações entre instituições e pesquisadores (AGUIAR, 2007; LOTUFO, 2009), bem como a estruturação dos Núcleos de Inovação Tecnológica (NITS). As atribuições gerais dos NITs concernem à gestão de políticas de inovação, considerando, ainda, alguns preceitos da lei de Propriedade Industrial (Lei $\mathrm{n}^{\circ}$. 9.279/96), o que torna os Núcleos de Inovação Tecnológica um dos 
principais intermediadores para a transferência de tecnologia entre as Instituições de Ciência e Tecnologia e a esfera empresarial (LOTUFO, 2009).

A respeito dessa temática, as contribuições de pesquisas já realizadas residem na identificação de fatores que facilitam e dificultam as relações (MORA-VALENTIN; MONTORO-SANCHEZ; GUERRAS-MARTIN, 2004), de elementos determinantes na seleção dos parceiros e na natureza dos resultados alcançados para cada tipo de cooperação. Depreende-se, aqui, que relações entre universidades e empresas (U-E), entre universidades e institutos de pesquisa (U-IP) e entre as próprias instituições acadêmicas (U-U) apresentam elementos que, se avaliados, podem oferecer novas possibilidades de pesquisas e melhor gerenciamento de projetos conjuntos, além de propiciarem que potenciais entraves sejam individuados.

Em face do exposto, neste artigo, buscou-se caracterizar as cooperações interinstitucionais, mormente, analisando-se os fatores que interferem nas cooperações em que a universidade se constitui como uma das partes envolvidas: os relacionamentos nos moldes Universidade-Empresa (U-E), Universidade-Universidade (U-U) e Universidade-Institutos de Pesquisa (U-IP). Assim, inicia-se o trabalho com a apresentação do referencial teórico referente à cooperação entre instituições. Em seguida, descreve-se a metodologia utilizada. Posteriormente, contextualiza-se o objeto de estudo e os casos analisados. Por fim, apresentam-se a análise e as considerações finais. 


\section{REFERENCIAL TEÓRICO}

\section{COOPERAÇÃO E INTERCÂMBIOS CIENTÍFICOS: CONTEXTUALIZAÇÃO DA LITERATURA}

Para que os processos de cooperação conjunta e de aprendizagem ocorram tanto na esfera empresarial como no âmbito acadêmico, torna-se necessário fomentar um ambiente propício às relações entre as várias instituições / atores que um sistema de inovação abrange (LUNDVALL, I992; ETZKOWITZ; LEYDESDORFF, 2000; ETZKOWITZ, 2002).

Sob essa ótica, o termo cooperação representa o vocábulo usualmente aplicado nos estudos que envolvem algum tipo de contato entre universidades, institutos de pesquisa e empresas. O Quadro I apresenta algumas das principais definições encontradas na literatura.

Quadro I Definição de cooperação com base na literatura

\begin{tabular}{|c|c|}
\hline Definição & Autor \\
\hline $\begin{array}{l}\text { [...] consórcio de pesquisa universidade-indústria e parcerias de pesquisa } \\
\text { - ou seja, relacionamentos colaborativos de pesquisa governados por } \\
\text { um acordo contratual, específico e detalhado e caracterizado pelo } \\
\text { planejamento, administração e implantação conjuntos de um significante } \\
\text { programa de pesquisa de longo prazo de interesse e benefício mútuo. }\end{array}$ & $\begin{array}{l}\text { Prager e Omen, (1980, } \\
\text { p. } 379)\end{array}$ \\
\hline $\begin{array}{l}\text { [...] modelo de arranjo interinstitucional entre organizações que têm } \\
\text { natureza fundamentalmente distinta; esse arranjo pode ter finalidades } \\
\text { variadas - desde interações tênues, como no oferecimento de estágios } \\
\text { profissionalizantes, até vínculos extensos e intensos, como nos grandes } \\
\text { programas de pesquisa cooperativa - e formatos bastante diversos. }\end{array}$ & Plonski (1994, p. 364) \\
\hline $\begin{array}{l}\text { Ligações que unem pesquisa básica (conduzida por universidades e } \\
\text { centros de pesquisa) com pesquisa aplicada (para o uso nas indústrias) } \\
\text { de tal forma que, como resultado da ação conjunta de ambas as partes, } \\
\text { sinergias podem ser criadas, que levam ao melhoramento do potencial } \\
\text { tecnológico dos parceiros que cooperam e, consequentemente, } \\
\text { aumentam o nível de competitividade dos países. }\end{array}$ & $\begin{array}{l}\text { Mora-Valentin, Montoro- } \\
\text { Sanchez e Guerras-Martin } \\
(2004, \text { p.17-18) }\end{array}$ \\
\hline $\begin{array}{l}\text { A cooperação U-E pode ser vista como instrumento de pesquisa } \\
\text { cooperativa entre empresas (públicas e privadas) e instituições } \\
\text { de pesquisa e universidades, em ação conjunta para desenvolver } \\
\text { novo conhecimento tecnológico que servirá para a ampliação do } \\
\text { conhecimento científico e para o desenvolvimento de novos produtos. } \\
\text { Permite a divisão de custos e riscos, além de ampliar a competitividade e } \\
\text { o conhecimento científico de um país. }\end{array}$ & $\begin{array}{l}\text { Segatto-Mendes e } \\
\text { Mendes }(2002 ; 2006)\end{array}$ \\
\hline
\end{tabular}


Numa comparação entre as definições apresentadas, nota-se a ênfase dada por Plonski (1995) às diferentes naturezas dos atores e, consequentemente, às variadas formas de interação deles. Mora-Valentin, Montoro-Sanchez e Guerras-Martin (2004) preocupam-se em mostrar que a cooperação busca a combinação e a complementação dos diferentes tipos de pesquisas para o alcance de melhores resultados. No conceito de cooperação de SegattoMendes e Mendes (2002), percebe-se maior destaque ao papel que esse tipo de relacionamento pode ter no desenvolvimento tecnológico e científico, que leva tanto ao avanço da ciência quanto ao desenvolvimento de novos produtos. Para Prager e Omen (1980), observa-se a questão do acordo contratual claro presente como o instrumento de governança da cooperação.

Sobre o papel da universidade, tem sido reconhecida sua posição enquanto agente de desenvolvimento econômico e regional em um contexto no qual o conhecimento tem-se tornado um "ativo", um "objeto de valor" cada vez mais importante, tendo em vista que essa instituição é reconhecidamente uma fonte de geração de conhecimento. De acordo com Etzkowitz e Leydesdorff (1997, p. I):

Canalizar fluxos de conhecimento em novas fontes de inovação tecnológica temse tornado uma tarefa acadêmica, mudando a estrutura e função da universidade. A realização dos benefícios desta potencial fonte ocorre por meio das inovações organizacionais tais como escritórios de transferência de tecnologia, instalações de incubadora e centros de pesquisa com participação industrial. A mudança na ênfase da única concentração na produção e disseminação de conhecimento para a transferência de tecnologia e a formação de empresas coloca a universidade em um novo alinhamento com o setor produtivo.

A extensa literatura que abrange o tema da cooperação, muitas vezes, toma o termo "universidade" para se referir a uma ampla gama de instituições de ensino e/ou pesquisa que realiza atividades cooperativas com o setor produtivo. O rótulo de "universidade" pode incluir instituições acadêmicas de Ensino Superior intensivas em pesquisa ou não, centros e institutos de pesquisa, as empresas juniores e até mesmo docentes que prestam algum tipo de consultoria individual (PLONSKI, I999; MORA-VALENTIN; MONTOROSANCHEZ; GUERRAS-MARTIN, 2004). Assim, com base no reconhecimento dessas 
novas atribuições da universidade, alguns dos autores que estudam o tema “cooperação U-E” revisitaram seus próprios conceitos. Nesse sentido, Plonski (I999, p. 5-6) versa que:

Os comentários iniciais focalizam a natureza dos sujeitos da cooperação. Pode ela envolver atores bastante distintos em cada uma de suas pontas. Assim, sob a denominação de empresa, geralmente pessoa jurídica, podem ser observadas empresas muito diferentes entre si, como uma transnacional de grande porte e tecnologia sofisticada na área de telecomunicações e uma microempresa que produz e comercializa velas decorativas artesanais para um mercado local [...]. No contexto de cooperação, o que se chama de empresa pode ser também uma pessoa física - como é o caso de um empreendedor potencial carente de apoio tecnológico e gerencial ou, então, de uma empresa informal, isto é, um negócio que opera sem estar na forma da lei.

Sob o rótulo de universidade pode ser encontrado, no contexto da cooperação com a empresa, um amplo leque de entidades de ensino e / ou pesquisa. Inclui, certamente, qualquer instituição de ensino superior - intensiva em pósgraduação e pesquisa avançada ou mera provedora de ensino de graduação; uma universidade, um centro universitário ou uma faculdade isolada; pública, comunitária ou privada e, neste caso, sem ou com fim lucrativo. [...] No entanto, o mesmo termo universidade aplica-se também, por vezes, a instituições de pesquisa não pertencentes a uma universidade, a fundações de direito privado conveniadas com uma instituição de ensino superior, a empresas juniores e, até mesmo, a docentes que se prestam a dar consultoria individual.

Essa multiplicidade de atores distintos que se abriga sob os nomes empresa e universidade podem estabelecer interações variadas, tanto no conteúdo transacional como na forma e, ainda, na estrutura de interface. (Grifo nosso).

Lotufo (2009, p.49) ainda aponta duas contribuições da universidade no fomento às capacidades inovativas do Brasil: "a formação de alunos para a inovação [...] e o aumento das chances de incorporação dos resultados das pesquisas e criações universitárias em benefício da sociedade”. Enquanto se tem ampliado as possibilidades de discussão na literatura sobre o relacionamento entre empresas, institutos de pesquisa e universidades, especialmente no tocante à forma, gestão e avaliação desses relacionamentos 
(FAULKNER; SENKER, VELHO, I995; SPENDER, I999; FAULKNER, SCHIBANNY, POLT, 200I; WRIGHT, 2008; COSTA; PORTO; FELDHAUS, 20IO), pOuca atenção tem sido atribuída às questões internas das universidades e às interações destas com outras e com os institutos de pesquisa, o que representa uma lacuna na literatura sobre essa temática. Entre tais questões, destaca-se o intercâmbio científico que, segundo Gatti (2005, p. I24-I25):

Processa-se não só por congressos e reuniões científicas de diversas naturezas, mas também por mecanismos como estágios, professores visitantes, desenvolvimento de projetos interinstitucionais, participações em redes de pesquisadores em temas correlatos, participações em grupos de pesquisa, etc. [...] esse intercâmbio pode desenvolver-se tanto em nível regional, nacional ou internacional, via programas básicos das agências de fomento à pesquisa, ou via programas de intercâmbio da Coordenação de Aperfeiçoamento de Pessoal de Nível Superior [...] e ainda por convênios bilaterais mantidos por algumas universidades com financiamento próprio.

Atualmente, algumas condições são essenciais para a realização de investigações e para o avanço do conhecimento científico. Destacam-se a necessidade de intercomunicação dos pesquisadores com seus pares; o trabalho em equipe; as redes de trocas de ideias; a disseminação de propostas e achados de investigação; e a consolidação de grupos de referência temática (ASSOCIAÇÃo NACIONAL DE PÓS-GRADUAÇÃo E PESQUISA EM EDUCAÇÃo [ANPED], I984; GATTI, 2005).

Nas ciências sociais aplicadas, o arcabouço teórico relacionado ao tema trata, predominantemente, das relações entre universidades e empresas, sendo que na busca por referencial não foi possível identificar maior número de referências acerca de cooperações entre universidades (Cooperação U/U) e entre universidades e institutos de pesquisa (Cooperação U/IPs), assim, denotando uma lacuna no conhecimento sobre o tema. Iniciativas para preencher esse espaço foram empreendidas por Rossoni e Guarido-Filho (2007; 2009), Rossoni e Hocayen-da-Silva (2008); Rossoni, Hocayen-da-Silva e Ferreira (2008), Melo, Crubellate e Rossoni (2009), que analisaram, por meio de métodos investigativos de natureza quantitativa, a cooperação entre instituições e pesquisadores em algumas áreas do campo da administração, 
e Dossa e Segatto (20I0), que procuraram caracterizar as cooperações entre universidades e institutos de pesquisa (U-IP) no setor agropecuário. Observouse, com base nesses avanços, um retrato do aumento das interações de pesquisadores de instituições distintas, parte em razão das alterações nos critérios de avaliação da CAPES, parte em razão do surgimento de novos grupos de pesquisa, amadurecimento daqueles já existentes e elevação do grau de especialização no campo científico (MELO; CRUBELLATE; ROSSONI, 2009).

Ao avaliar publicações dos últimos sete anos que abrangem a questão das cooperações, estudos conjuntos e interação da universidade com outros atores, observou-se a seguinte distribuição nos principais periódicos nacionais apresentada no Quadro 2:

Quadro 2 Artigos que discorrem sobre a cooperação nos moldes U-E; U-IP ou U-U

\begin{tabular}{|c|c|c|}
\hline Periódico & $\begin{array}{l}\text { Número de } \\
\text { artigos }\end{array}$ & Autores \\
\hline RAM & 2 artigos & Melo, Crubellate e Rossoni (2009); Rossoni (2014) \\
\hline RAC & 6 artigos & $\begin{array}{l}\text { Gimarães et al. (2009); Rossoni e Guarido-Filho (2009); Cruz e } \\
\text { Segatto (2009); Costa, Porto e Feldhaus (2010); Dias e Porto (2013); } \\
\text { Mendes-da-Silva, Onusic e Giglio (2013) }\end{array}$ \\
\hline BAR & Sem artigos & - \\
\hline RAE & Sem artigos & - \\
\hline RAI & 11 artigos & $\begin{array}{l}\text { Li, Ferreira e Serra (2009); Pereira et al. (2009); Araujo e Teixeira } \\
\text { (2010); Costa, Porto e Plonski (2010); Lopes e Judice (2010); Noveli e } \\
\text { Segatto (2012); Villela e Almeida (2012); Vitorino Filho et al. (2012); } \\
\text { Alves e Pereira (2013); Mussi e Segatto (2013) }\end{array}$ \\
\hline RAUSP & Sem artigos & - \\
\hline RBI & 5 artigos & $\begin{array}{l}\text { Burcharth (2011); Alvarez, Kannebley Junior e Carolo (2013); Rapini, } \\
\text { Oliveira e Silva Neto (2014); Schaeffer, Rufonni e Puffal (2015); Britto } \\
\text { et al. (2015) }\end{array}$ \\
\hline
\end{tabular}

Assim, o referencial apresentado se centra, principalmente, nas contribuições relativas às cooperações interinstitucionais de modo mais amplo, sendo que conceitos comuns ou taxonomias já conhecidos e que atendem às diversas relações interinstitucionais são adotados para o suporte teórico necessário ao estudo. 


\section{MECANISMOS DE INTERAÇÃO}

Várias são as formas de interações institucionais contempladas pela literatura. Bonaccorsi e Piccaluga (I994) mencionam seis possibilidades de relações interinstitucionais, conforme exposto no Quadro 3:

\section{Quadro 3 Formas de relações e mecanismos na interação U-E}

\begin{tabular}{|c|c|c|}
\hline $\begin{array}{l}\text { Formas de } \\
\text { relaçōes }\end{array}$ & Descrição & Exemplos \\
\hline $\begin{array}{l}\text { Relações pessoais } \\
\text { informais }\end{array}$ & $\begin{array}{l}\text { Ocorrem quando a empresa e uma } \\
\text { pessoa da universidade efetuam } \\
\text { trocas sem que seja elaborado } \\
\text { qualquer acordo formal que envolva a } \\
\text { universidade. }\end{array}$ & $\begin{array}{l}\text { (1) Consultoria individual; (2) workshops } \\
\text { informais; ( } 3 \text { ) spin-offs acadêmicos; (4) } \\
\text { publicações de resultados de pesquisa }\end{array}$ \\
\hline $\begin{array}{l}\text { Relações pessoais } \\
\text { formais }\end{array}$ & $\begin{array}{l}\text { São como as relações pessoais } \\
\text { informais, mas com a presença de } \\
\text { acordos formalizados. }\end{array}$ & $\begin{array}{l}\text { (1) bolsas de estudo e apoio à pós- } \\
\text { graduação; (2) estágios de alunos e } \\
\text { cursos sandwich; (3) períodos sabáticos } \\
\text { para professores; (4) intercâmbio de } \\
\text { pessoal }\end{array}$ \\
\hline Terceira parte & $\begin{array}{l}\text { Existe um grupo intermediário. Essas } \\
\text { associações que intermediarão as } \\
\text { relações podem estar dentro da } \\
\text { universidade, ser externas ou estar em } \\
\text { posições intermediárias. }\end{array}$ & $\begin{array}{l}\text { (1) escritórios especializados em } \\
\text { intermediação; ( } 2 \text { ) associações } \\
\text { industriais; (3) institutos de pesquisa } \\
\text { aplicada; (4) escritórios de assistência } \\
\text { geral; (5) fundações universitárias }\end{array}$ \\
\hline $\begin{array}{l}\text { Acordos formais } \\
\text { com alvos } \\
\text { definidos }\end{array}$ & $\begin{array}{l}\text { Relações em que ocorre tanto a } \\
\text { formalização do acordo como } \\
\text { também a definição dos objetivos } \\
\text { específicos de colaboração desde o } \\
\text { início. }\end{array}$ & $\begin{array}{l}\text { (1) pesquisa e serviços contratados; } \\
\text { (2) treinamento de funcionários das } \\
\text { empresas; (3) treinamento on the } \\
\text { job para estudantes; (4) projetos de } \\
\text { pesquisa cooperativa }\end{array}$ \\
\hline $\begin{array}{l}\text { Acordos formais } \\
\text { sem alvos } \\
\text { definidos }\end{array}$ & $\begin{array}{l}\text { Acordos formalizados como no caso } \\
\text { anterior, mas cujas relações possuem } \\
\text { maior amplitude com objetivos } \\
\text { estratégicos e de longo prazo. }\end{array}$ & $\begin{array}{l}\text { (1) convênios "guarda-chuva"; ( } 2 \text { ) } \\
\text { patrocínio industrial de P\&D em } \\
\text { departamentos da universidade; } \\
\text { (3) doações e auxílio para pesquisa, } \\
\text { genéricos ou para departamentos } \\
\text { definidos }\end{array}$ \\
\hline $\begin{array}{l}\text { Criação de } \\
\text { estruturas } \\
\text { focalizadas }\end{array}$ & $\begin{array}{l}\text { Iniciativas de pesquisa conduzidas } \\
\text { conjuntamente pela indústria } \\
\text { e universidade em estruturas } \\
\text { permanentes específicas criadas para } \\
\text { tal propósito, entre outros. }\end{array}$ & $\begin{array}{l}\text { (1) contratos de associação; ( } 2 \text { ) } \\
\text { consórcios de pesquisa U-E; ( } 3 \text { ) } \\
\text { incubadoras de empresas; (4) parques } \\
\text { tecnológicos }\end{array}$ \\
\hline
\end{tabular}

Fonte: Adaptado de Bonaccorsi e Piccaluga (1994 apud segatto-mendes, 2001, p.70).

Cabe ressaltar, conforme afirma Segatto (1996), que a escolha do instrumento de cooperação mais adequado dependerá, principalmente, da posição e 
dos objetivos dos participantes no processo, para tanto, contemplando a maleabilidade e a adequação correspondente ao tipo de relação a ser estabelecida. Ademais, independentemente do tipo da relação por meio da qual se desenvolve a cooperação, Porto (2000) recomenda, sobremaneira, a necessidade da existência de um gerenciamento do processo, considerando que a complexidade das interações se eleva conforme o tipo das relações apresentadas no Quadro 3.

Nesse contexto, as estruturas de interface constituem mecanismos intermediários úteis para promover e facilitar as cooperações. Para Segatto (1996), esses intermediadores caracterizam-se como agentes e organizações, sendo que os acordos cooperativos se utilizam deles para a administração de certos aspectos da interação, como o controle de recursos, a divulgação de linhas de pesquisa, o contato com instituições parceiras potenciais e a facilitação e manutenção da comunicação entre as partes. Essas funções podem ser executadas por órgãos como fundações, escritórios de transferência de tecnologia, pró-reitorias ou outros.

Vale lembrar que, no Brasil, os escritórios de transferência de tecnologia ainda não foram beneficiados com uma legislação particular que permitisse uma delimitação de suas funções (SANTOS; SOLLEIRO; LAHORgUe, 2004). Ainda no âmbito nacional, destaque deve ser atribuído à lei de inovação (Lei ${ }^{\circ}$. I0.973, 2004), regulamentada pelo Decreto $n^{\circ} .5 .563$, de II de outubro de 2005, que define medidas de estímulo à inovação e à pesquisa científica e tecnológica no ambiente produtivo, objetivando a capacitação e o alcance da autonomia tecnológica, e ao desenvolvimento industrial do país. Entretanto Lotufo (2009) reconhece que o país ainda se encontra distante dos demais países emergentes quando são avaliados os pedidos de patentes e a correspondente questão da inovação. Outros pontos de destaque se referem aos gastos em Pesquisa e Desenvolvimento (P\&D), considerados muito inferiores aos dispêndios de países desenvolvidos e à estrutura desses desembolsos, em sua maioria, de responsabilidade do setor público, fato que não se verifica em países desenvolvidos, onde o setor privado assume majoritariamente essa atribuição (BOHER et al., 2007). 


\section{FATORES MOTIVADORES PARA COOPERAÇÕES}

A conclusão de um processo cooperativo pode gerar vários resultados, visto que a motivação das partes envolvidas pode ser calcada em fatores distintos. Mora-Valentin, Montoro-Sanchez e Guerras-Martin (2004) apresentam alguns fatores-chave que podem fomentar a cooperação. Entre os fatores motivacionais, destacam-se as ligações organizacionais, a confiança entre as partes, o fluxo de comunicação, o comprometimento e as relações de dependência entre os envolvidos, tendo em vista os objetivos fixados para a interação.

Segatto (I996), com base nos estudos de Bonaccorsi e Piccaluga (I994), apresenta alguns dos motivadores que influenciam na decisão das universidades de estabelecer relações de cooperação. Entre esses motivadores, citam-se: falta de fontes financiadoras de pesquisa; carência de equipamentos e/ ou materiais para laboratório; possibilidade de geração de renda adicional para o pesquisador e para o centro de pesquisa; aumento do prestígio institucional; difusão do conhecimento e expansão das perspectivas profissionais do pesquisador. Mussi e Segatto (20I3), avaliando as motivações para a cooperação em dez projetos de pesquisa envolvendo universidades e institutos de pesquisa, identificaram como principais motivadores: fortalecimento do grupo de pesquisa e do nome da instituição; auxílio financeiro a projetos de pesquisa; complementação a projetos já iniciados; possibilidade da criação de patentes e melhora na infraestrutura da instituição e/ ou do grupo de pesquisa. A próxima seção complementa a discussão apresentando alguns facilitadores e dificultadores das cooperações.

\section{DIFICULTADORES E FACILITADORES À COOPERAÇÃO}

São diversos os dificultadores e os facilitadores que causam interferência no processo de cooperação, independentemente da disposição das partes envolvidas. Questões como grau de incerteza e duração dos projetos (CYERT; GOODMAN, I997); discrepância nos objetivos (SEGATTO, I996); complexidade do contrato cooperativo (PORTO; PLONSKI, 2000; SEGATTO-MENDES; SBRAGIA, 2002), entre outras, denotam a multiplicidade de elementos que podem, circunstancialmente, constituir barreiras ou mesmo ser facilitadores da cooperação. 
Becker, Jornada e Ritter (1996) sugerem um arcabouço conceitual para a compreensão da motivação de cooperação. Com ênfase nas universidades, são discutidos o nível de atuação e as motivações institucional e individual. Cita-se, por exemplo, a complexidade da organização acadêmica, que preserva fronteiras rígidas entre diferentes faculdades e/ou departamentos universitários, desse modo, proporcionando certa fragmentação do seu espaço e limitando interação interna e o contato entre os diferentes setores da universidade (coordenações e pró-reitorias, por exemplo). Sugere-se que são encontradas diversas restrições quando se deseja obter cooperação por meio dessas fronteiras.

Como facilitador de relações cooperativas, Aguiar (2007) identificou, empiricamente, numa pesquisa desenvolvida no estado de Minas Gerais, que:

- Os $62,05 \%$ dos casos de formação de redes e projetos cooperativos surgiram como resposta às ações promovidas pelas agências de financiamento, especialmente por meio dos editais.

- Havia elevada participação (8I,2\%) de instituições governamentais de financiamento.

- Grande parte dos pesquisadores acredita que as pesquisas e os projetos executados em conjunto, na forma de cooperação, são positivos e benéficos.

Na pesquisa de Aguiar (2007), verificou-se, em uma perspectiva quantitativa, a participação das agências de financiamento nos projetos cooperativos. Tais mecanismos podem atenuar a intensidade com que eventuais barreiras incidam sobre os agentes desse processo, desse modo, melhorando, também, os resultados da cooperação, pois algumas dessas agências lançam editais com regras e prazos já fixados. No tocante às instituições facilitadoras da cooperação interinstitucional, cabe destacar o papel da Coordenação de Aperfeiçoamento de Pessoal de Nível Superior (CAPES), do Conselho Nacional de Desenvolvimento Científico e Tecnológico (CNPQ) e da Financiadora de Estudos e Projetos (FINEP), além das fundações estaduais que sistematicamente fomentam, com base em seus editais, a interação de instituições de ensino e pesquisa. 
Com o intuito de ampliar os cursos e as atividades da pós-graduação e intensificar a interação de instituições brasileiras e estrangeiras, a modalidade de interação denominada de "professor-visitante" tem sido regularmente contemplada nesses editais (COORDENAÇÃO DE APERFEIÇOAMENTO DE PESSOAL DE NÍVEL SUPERIOR [CAPES], 20I2a). Nessa modalidade, observa-se o escopo de atuação do professor visitante por meio das seguintes possibilidades: (I) orientações de graduação, mestrado e doutorado; (2) publicação de trabalhos em congressos e periódicos nacionais e internacionais; (3) publicação de livros; (4) desenvolvimento de softwares e outros materiais; (5) organização de eventos; (6) cursos / disciplinas oferecidas e suas respectivas contribuições inovadoras; e (7) prêmios recebidos (CAPES, 20I2b).

A literatura sugere, ainda, um arcabouço conceitual para melhor compreensão da motivação e dos entraves para a interação da pesquisa acadêmica e a indústria. Com relação à universidade, são discutidos os níveis de atuação institucional e o individual. Pelo lado da indústria, as associações / federações e firmas individuais representam os níveis analisados - vide Quadro 4. 


\section{Quadro 4 Arcabouço conceitual da universidade e da empresa}

\begin{tabular}{|c|c|c|}
\hline \multirow[t]{2}{*}{ Universidade } & Nível institucional & $\begin{array}{l}\text { (1) Complexidade da organização acadêmica, que } \\
\text { preserva fronteiras rígidas entre as diferentes faculdades } \\
\text { ou departamentos universitários, proporcionando certa } \\
\text { fragmentação do seu espaço e limitando a interação interna e o } \\
\text { contato entre os diferentes setores da universidade. Sugere-se } \\
\text { que são encontradas diversas restrições quando se deseja obter } \\
\text { cooperação com base nessas fronteiras. (2) Ocorrência de certa } \\
\text { polarização do campo de ação institucional por programas } \\
\text { de fomento que são controlados, de forma centralizada, } \\
\text { pelos governos federais ou estaduais, que não induzem } \\
\text { estratégias que propiciem o fortalecimento de cooperação } \\
\text { efetiva entre diferentes departamentos. (3) O jogo do poder } \\
\text { entre as instituições versa quase que predominantemente a } \\
\text { maximização dos possíveis incentivos financeiros provenientes } \\
\text { dos órgãos de fomento que estabelecem critérios que focam } \\
\text { o aumento da produção de documentos técnico-científicos } \\
\text { ou o número de cursos de pós-graduação. Nesse contexto, a } \\
\text { interação com a indústria não é favorecida, uma vez que } \\
\text { os critérios de avaliação de tais entidades de fomento não } \\
\text { privilegiam os trabalhos não acadêmicos. }\end{array}$ \\
\hline & Nível individual & $\begin{array}{l}\text { (1) As relações de poder no meio acadêmico são balizadas } \\
\text { por fatores de caráter técnico e político. Quanto aos últimos, } \\
\text { destaca-se a posição do indivíduo na instituição, sua rede de } \\
\text { contatos interinstitucionais, participação em comitês, órgãos } \\
\text { assessores, organização de eventos técnico-científicos. Com } \\
\text { relação aos primeiros, ressalta-se a titulação acadêmica e os } \\
\text { trabalhos científicos. Assim sendo, a interação com a indústria } \\
\text { não é valorizada. }\end{array}$ \\
\hline \multirow[t]{2}{*}{ Indústria } & $\begin{array}{l}\text { Associações } \\
\text { comerciais e } \\
\text { federações }\end{array}$ & $\begin{array}{l}\text { (1) As associações comerciais, as federações das indústrias e } \\
\text { os sindicatos representam os interesses dos seus respectivos } \\
\text { setores perante o governo e procuram manter, com este, laços } \\
\text { apertados, assim, influenciando políticas públicas de tarifação, } \\
\text { planos de desenvolvimento industrial, etc. A globalização da } \\
\text { economia tem trazido muitas mudanças nas regras desse jogo } \\
\text { de poder, e, portanto, a importância do desenvolvimento } \\
\text { tecnológico deve mudar o centro de ação desse segmento. } \\
\text { Dessa forma, é possível que novos mecanismos de fomento à } \\
\text { interação de U-IPs-E surjam para suprir eventuais defasagens } \\
\text { tecnológicas e de conhecimento das empresas que competem } \\
\text { globalmente. }\end{array}$ \\
\hline & Firmas individuais & $\begin{array}{l}\text { Conquanto grande parte das firmas esteja focalizada no lucro } \\
\text { imediato, a intensa competição e a formação de redes de } \\
\text { empresas propiciaram um ambiente favorável para a busca de } \\
\text { novas tecnologias, sendo que a esfera pública (universidades } \\
\text { e IPs) pode auxiliar nessa carência. Contudo é ressaltada a } \\
\text { ausência de um agente que facilite tal aproximação e interação. }\end{array}$ \\
\hline
\end{tabular}

Fonte: Adaptado de Becker, Jornada e Ritter (1996). 


\section{METODOLOGIA}

Esta pesquisa classifica-se como mista, pois concilia métodos de pesquisa qualitativos e quantitativos (MALHOTRA, 200I). McDaniel e Gates (2003) mencionam a importância de estudos que conciliem tais métodos, pois estes proporcionam maior compreensão da realidade, geralmente, extraindo dados mais reveladores. A pesquisa tem perspectiva temporal seccional e focaliza um nível de análise organizacional - na fase qualitativa - e individual, na fase quantitativa.

Na etapa qualitativa, de natureza exploratória, objetivou-se adquirir maior conhecimento sobre o fenômeno investigado (YIN, 200I). Dessa forma, foram entrevistados, por intermédio de um roteiro semiestruturado, 23 professores/pesquisadores participantes de dez projetos de cooperações interinstitucionais em universidades e institutos de pesquisa localizados no estado do Paraná.

O critério para seleção dos entrevistados foi o grau de envolvimento com a cooperação, assim: coordenadores de projetos de cooperação técnica; professores visitantes; outros professores fortemente envolvidos. Posteriormente à coleta de dados, executou-se uma apreciação do conteúdo de forma sistemática. Concomitantemente às entrevistas, coletaram-se dados secundários, analisando-se os materiais que originaram os projetos de cooperação estudados. Também, foram realizadas oito visitas técnicas às instituições onde as atividades estavam sendo desenvolvidas, totalizando, aproximadamente, 96 horas de observação não participante. Dos resultados dessa etapa, foi proposto um modelo de análise das cooperações interinstitucionais (MUSSI; SEGATTO, 20I3).

$\mathrm{Na}$ etapa quantitativa, de natureza descritiva, o instrumento de coleta utilizado foi concebido com base nos achados na fase qualitativa, bem como daquelas contribuições consolidadas pela literatura, passando pelo processo de validação de face (FINK, I995), em que dois pares acadêmicos avaliaram inconsistências e equívocos observados do instrumento. Na sequência, foram realizados pré-testes presenciais e virtuais. O Quadro 5 sintetiza o conteúdo proposto na pesquisa. 
Quadro 5 Síntese do conteúdo da pesquisa

\begin{tabular}{|c|c|c|}
\hline Construto & Variáveis & Autores \\
\hline $\begin{array}{l}\text { Instrumento de } \\
\text { cooperação }\end{array}$ & $\begin{array}{l}\text { Relações pessoais informais; relações pessoais com a } \\
\text { presença de acordos formalizados; terceira parte; acordos } \\
\text { formais com alvos definidos (acordos de cooperação/ } \\
\text { contratos/convênios); acordos formais sem alvos } \\
\text { definidos; estruturas focalizadas. }\end{array}$ & $\begin{array}{l}\text { Bonaccorsi e Piccaluga (1994); } \\
\text { Segatto (1996); Costa, Porto e } \\
\text { Feldhaus (2010) }\end{array}$ \\
\hline $\begin{array}{l}\text { Motivadores para a } \\
\text { cooperação }\end{array}$ & $\begin{array}{l}\text { Fortalecimento/consolidação do grupo de pesquisa e da } \\
\text { instituição; auxílio financeiro para projetos de pesquisa; } \\
\text { complementação/continuidade de projetos de pesquisa } \\
\text { já iniciados; possibilidade de criação de patentes; melhora } \\
\text { na infraestrutura do grupo de pesquisa e da instituição; } \\
\text { troca de informações; educação; ideias para novas } \\
\text { pesquisas. }\end{array}$ & $\begin{array}{l}\text { Prager e Omenn (1980); } \\
\text { Bonaccorsi e Piccaluga (1994); } \\
\text { Segatto (1996); Mora-Valentin, } \\
\text { Montoro-Sanchez e Guerras- } \\
\text { Martin (2004); Arza, (2010); } \\
\text { Shima e Scatolin (2011); Porto } \\
\text { et al., (2011); Mussi e Segatto } \\
\text { (2013) }\end{array}$ \\
\hline $\begin{array}{l}\text { Determinantes da } \\
\text { escolha do parceiro }\end{array}$ & $\begin{array}{l}\text { Credibilidade quanto à capacidade técnica; às } \\
\text { experiências anteriores bem-sucedidas; à compatibilidade } \\
\text { de interesses; à disponibilidade para atender ao } \\
\text { cronograma. }\end{array}$ & $\begin{array}{l}\text { Segatto (1996); Mussi e Segatto } \\
\text { (2013) }\end{array}$ \\
\hline $\begin{array}{l}\text { Dificultadores para } \\
\text { a cooperação }\end{array}$ & $\begin{array}{l}\text { Excesso de burocracia para comprovação de execução } \\
\text { das despesas; excesso de burocracia por parte da } \\
\text { universidade ou do instituto de pesquisa; excesso de } \\
\text { controles da empresa; falta de recursos humanos para a } \\
\text { execução dos projetos; falta de apoio das pró-reitorias; } \\
\text { falta de recursos dos grupos de pesquisa; atraso nos } \\
\text { pagamentos; dificuldades para cumprimento do } \\
\text { cronograma de execução dos projetos conjuntos. }\end{array}$ & $\begin{array}{l}\text { Becker, Jornada e Ritter } \\
\text { (1996); Segatto (1996); Cyerte } \\
\text { Goodman (1997); Mota (1999); } \\
\text { Porto e Plonski (2000); Segatto- } \\
\text { mendes e Sbragia (2002); Aguiar } \\
\text { (2007); Mussi e Segatto (2013); } \\
\text { Freitas, Marques e Silva (2013) }\end{array}$ \\
\hline $\begin{array}{l}\text { Facilitadores para a } \\
\text { cooperação }\end{array}$ & $\begin{array}{l}\text { Compartilhamento de objetivos e interesses; } \\
\text { conhecimento prévio do parceiro em virtude de } \\
\text { experiências anteriores; parceiro domina a área de } \\
\text { conhecimento técnico que atua; custos viáveis para } \\
\text { execução dos projetos. }\end{array}$ & \\
\hline $\begin{array}{l}\text { Resultados/ } \\
\text { contribuições da } \\
\text { cooperação }\end{array}$ & $\begin{array}{l}\text { Publicações científicas; teses e dissertações; registro de } \\
\text { patentes; melhora da infraestrutura física dos grupos } \\
\text { de pesquisa; contribuições às comunidades locais; } \\
\text { transferência de conhecimento; os novos projetos de } \\
\text { pesquisa. }\end{array}$ & $\begin{array}{l}\text { Faulkner, Senker e Velho } \\
\text { (1995); Segatto (1996); Cyert } \\
\text { e Goodman (1997); Meyer- } \\
\text { Krahmer e Schmoch (1998); } \\
\text { Daghfous (2003); Rapini et al. } \\
\text { (2009); Fernandes et al. (2010) }\end{array}$ \\
\hline
\end{tabular}

A coleta de dados se deu por meio de uma survey com os grupos de pesquisa, localizados em universidades do estado do Paraná e pertencentes aos diversos campos do conhecimento. Para a execução da investigação, contataram-se, por telefone, os grupos de pesquisa e foi enviado, por e-mail, o arquivo com o questionário. Foram enviados 92 questionários, dos quais retornaram $64(69,56 \%$ de retorno). Para a tabulação dos dados, utilizou-se o Microsoft Excel e, para análise, o pacote estatístico spss. 


\section{APRESENTAÇÃO DOS DADOS}

A análise descritiva dos dados $(\mathrm{N}=64)$ revelou que $76 \%$ dos respondentes pertenciam a programas de pesquisa de universidades públicas e $24 \%$ pertenciam a programas de pesquisa de universidades privadas. Dos processos de cooperação analisados, $45 \%$ corresponderam a interações do tipo "universidade-empresa (U-E)", $37 \%$ representaram interações do tipo "universidade-universidade (U-U)" e I $8 \%$ consistiram em interações entre "universidades-institutos de pesquisa (U-IP)". As áreas de conhecimento nas quais as cooperações eram realizadas estão dispostas conforme apresentado na Tabela I.

Tabela I Áreas de conhecimento contempladas na pesquisa

\begin{tabular}{|ll|}
\hline Área do conhecimento & Número de casos \\
\hline Ciências Agrárias & 7 \\
\hline Ciências Sociais & 7 \\
\hline Ciências Humanas & 9 \\
\hline Ciências Sociais Aplicadas & 9 \\
\hline Ciências Biológicas & 10 \\
\hline Ciências Exatas & 22 \\
\hline Total & $\mathbf{6 4}$ \\
\hline
\end{tabular}

Para verificar a distribuição normal dos dados, utilizou-se o teste de KolmogorovSmirnov. Os resultados foram os seguintes: na dimensão "motivadores do processo de cooperação", D (64) 0,26 p<0,05; na dimensão "dificultadores ao processo de cooperação", D (64) 0,16 p <0,05; na dimensão "determinantes da escolha do parceiro para a cooperação", D (64) 0,243 p<0,05; na dimensão "facilitadores do processo de cooperação", D (64) o,I77 p<0,05; e, na dimensão "contribuições/ resultados do processo de cooperação", D (64) 0,22 p<0,05. Assim, concluiu-se que os dados se apresentam como uma distribuição não normal.

Para verificação da formação dos construtos mensurados, esses foram submetidos à Análise Fatorial Exploratória (AFE). Com exceção do construto "facilitadores ao processo de cooperação", que indicou a formação de dois fatores, a AFE sugeriu que os demais construtos se comportam como prevê 
a literatura. Assim mesmo, o modelo foi testado conforme previa a teoria em voga, ou seja, forçando que os indicadores formassem um construto a priori (HAIr et al., 2005). Para testar a consistência interna de cada construto, foram mensurados os Alphas de Cronbach ( $\alpha$ ) para cada dimensão. Com relação às quatro dimensões levantadas pelo instrumento de pesquisa, na dimensão "facilitadores do processo de cooperação" $(\alpha=0,529)$ sugeriu-se o descarte por não apresentar consistência interna satisfatória.

- A dimensão "motivadores do processo de cooperaçãa" apresentou consistência interna satisfatória $(\alpha=0,617)$ quando medida pelos seguintes itens:

a. auxilio financeiro em projetos de pesquisa;

b. melhora na infraestrutura da instituição.

- A dimensão "dificultadores ao processo de cooperação" apresentou consistência interna satisfatória $(\alpha=0,632)$ quando medida pelos seguintes itens:

a. falta de apoio das pró-reitorias;

b. falta de recursos (humanos e financeiros) dos programas de pesquisa;

c. atraso nos pagamentos;

d. excesso de burocracia na comprovação de despesas

- A dimensão "determinantes da escolha do parceiro para a cooperação" apresentou, inicialmente, consistência interna satisfatória $(\alpha=0,7 \mathrm{II})$ quando medida pelos seguintes itens:

a. credibilidade da instituição parceira reconhecida pela capacidade técnica na área do projeto;

b. existência de experiências anteriores bem-sucedidas entre os parceiros;

c. disponibilidade do parceiro para atender o cronograma do projeto a ser desenvolvido.

Posteriormente, a fim de confirmar a existência de associação entre os tipos de cooperação (U-U; U-E; U-IP) e os fatores motivadores, dificultadores e elementos determinantes da cooperação, realizou-se o teste Qui-quadrado. Nesse contexto, as hipóteses formuladas foram:

- $\mathrm{H}_{0}$ : não existe associação estatisticamente significativa entre os elementos e os tipos de cooperação analisados; 
- $\mathrm{H}_{\mathrm{r}}$ : existe associação estatisticamente significativa entre os elementos e os tipos de cooperação analisados.

Quadro 6 Teste Qui-quadrado nas variáveis que compõem cada dimensão da pesquisa

\begin{tabular}{|c|c|}
\hline $\begin{array}{l}\text { Motivador: auxílio } \\
\text { financeiro em projetos } \\
\text { de pesquisa }\end{array}$ & $\begin{array}{l}\text { - Interfere significativamente na motivação de cooperar com Sig=0,05 } \\
\text { empresas. } \\
\text {-Interfere pouco na motivação de cooperação entre universidades. } \\
\text { - Interfere razoavelmente na motivação de cooperar com institutos } \\
\text { de pesquisa. }\end{array}$ \\
\hline $\begin{array}{l}\text { Motivador: melhora } \\
\text { na infraestrutura da } \\
\text { instituição }\end{array}$ & $\begin{array}{l}\text { - Interfere significativamente na motivação de cooperar com Sig<0,05 } \\
\text { empresas. } \\
\text { - Interfere pouco na motivação de cooperação entre universidades. } \\
\text { - Interfere razoavelmente na motivação de cooperar com institutos } \\
\text { de pesquisa. }\end{array}$ \\
\hline $\begin{array}{l}\text { Dificultador: excesso } \\
\text { de burocracia na } \\
\text { comprovação de } \\
\text { despesas }\end{array}$ & $\begin{array}{l}\text { - Interfere significativamente na motivação de cooperar com Sig<0,05 } \\
\text { empresas. } \\
\text { - Não interfere na motivação de cooperação entre universidades. } \\
\text { - Interfere razoavelmente na motivação de cooperar com institutos } \\
\text { de pesquisa. }\end{array}$ \\
\hline $\begin{array}{l}\text { Dificultador: falta de } \\
\text { recursos humanos para } \\
\text { executar o projeto }\end{array}$ & - Interfere razoavelmente em todos os tipos de cooperação. \\
\hline $\begin{array}{l}\text { Determinante: } \\
\text { credibilidade da } \\
\text { instituição }\end{array}$ & $\begin{array}{l}\text { - Interfere significativamente na motivação de cooperar com Sig<0,05 } \\
\text { universidades e institutos de pesquisa. } \\
\text { - Interfere pouco na motivação de cooperar com empresas. }\end{array}$ \\
\hline $\begin{array}{l}\text { Determinante: } \\
\text { experiências anteriores } \\
\text { bem-sucedidas }\end{array}$ & $\begin{array}{l}\text { - Interfere significativamente na motivação de cooperar com Sig<0,05 } \\
\text { universidades e institutos de pesquisa. } \\
\text { - Interfere pouco na motivação de cooperar com empresas. }\end{array}$ \\
\hline
\end{tabular}

Buscou-se, também, verificar a associação entre os tipos de cooperação observados na pesquisa e a área de conhecimento na qual a cooperação estava sendo realizada. Foram trabalhadas as hipóteses de que não existe associação $\left(\mathrm{H}_{\mathrm{o}}\right)$ e de que a associação é existente $\left(\mathrm{H}_{\mathrm{r}}\right)$ (vide Tabela 2).

Tabela 2 Avaliação entre tipos de cooperação e áreas de conhecimento

\begin{tabular}{|llll|}
\hline & Valor & df & Sig. Assint (2 lados) \\
\hline Qui-quadrado de Pearson & $38,796^{\mathrm{a}}$ & 24 & 0,029 \\
\hline Razão de verossimilhança & 38,931 & 24 & 0,28 \\
\hline Número de casos válidos & 64 & & \\
\hline
\end{tabular}


Verificou-se que:

- na cooperação U-E, predominam as pesquisas na área de ciências exatas e de ciências biológicas;

- na cooperação U-U, predominam as pesquisas na área de ciências exatas, sociais aplicadas e sociais;

- na cooperação U-IP, predominam as pesquisas na área de ciências exatas. Por fim, tratou-se, ainda, de verificar a associação entre os tipos de cooperação observados na pesquisa e os mecanismos (instrumentos) que regiam a relação de cooperação. Foram trabalhadas as hipóteses de que não existe associação $\left(\mathrm{H}_{0}\right)$ e de que a associação é existente $\left(\mathrm{H}_{\mathrm{I}}\right)$ (vide Tabela 3).

Tabela 3 Avaliação entre tipos de cooperação e instrumentos contratuais

\begin{tabular}{|llll|}
\hline & Valor & df & Sig. Assint(2 lados) \\
\hline Qui-quadrado & $43,175^{\mathrm{a}}$ & 12 & 0,000 \\
\hline Razão de verossimilhança & 42,943 & 12 & 0,000 \\
\hline No de casos válidos & 64 & & \\
\hline
\end{tabular}

Verificou-se que:

- na cooperação U-E, predominam o convênio e o acordo formal de cooperação como os principais mecanismos;

- na cooperação U-U, predominam o acordo de cooperação formal e o acordo informal como os principais mecanismos;

- na cooperação U-IP, o acordo formal é o principal mecanismo que rege a interação.

\section{RESULTADOS/CONTRIBUIÇÕES DOS PROCESSOS DE COOPERAÇÃO}

Quando analisadas as contribuições que os processos de cooperação trouxeram para as instituições respondentes da presente pesquisa, observouse que - na perspectiva da universidade - as publicações representam o item mais lembrado quando a interação se dá com uma empresa ou instituto de pesquisa. A respeito das interações com organizações empresariais, destacase a possibilidade de melhorar a infraestrutura da instituição no sentido de modernizar laboratórios, adquirir equipamentos que - sem a cooperação 
- dificilmente poderiam ser comprados. Tal constatação foi corroborada nas visitas técnicas, quando se conheceram algumas estruturas precárias de instalação e de equipamentos, que não poderiam ser repostos/substituídos exclusivamente com recursos financeiros da universidade.

Nas cooperações entre universidades, chama atenção a preocupação dos respondentes em assinalar a transferência de conhecimento entre instituições (programas de pesquisa ou mesmo entre pesquisadores) como a principal contribuição, denotando o cuidado em qualificar novos pesquisadores, docentes e alunos. Durante as entrevistas, ressaltou-se a preocupação em trazer pesquisadores externos para suprirem lacunas de conhecimento nos grupos de pesquisa. Cabe ressaltar, também, o desenvolvimento de teses e dissertações como um dos frutos do trabalho conjunto na esfera acadêmica. Muitos dos pesquisadores externos contribuíam como coorientadores. Esses achados vão ao encontro das constatações da literatura especializada atinente à capacitação e à qualificação de pesquisadores no Brasil.

O sistema de pós-graduação tem propiciado um crescente número de mestres e doutores, em um leque diversificado do conhecimento. A associação entre pósgraduação e pesquisa possibilitou a evolução positiva de diversos indicadores, entre eles as publicações científicas [...] Estes fatores contribuíram para um aumento exponencial da quantidade e qualidade do desenvolvimento científico do País, medidos pelas publicações indexadas e a formação de doutores e mestres (LOTUFO, 2009, p.47).

No tocante às cooperações com institutos de pesquisa, um dos itens mais citados refere-se às contribuições potenciais às comunidades locais. Nesse ponto, sobressaíram-se as pesquisas na área agrícola, como a cultura de uva, mandioca e milho no estado do Paraná. A Tabela 4 sintetiza os itens mais mencionados.

Tabela 4 Avaliação entre tipos de cooperação e principais contribuições/resultados

\begin{tabular}{|llll|}
\hline U-E & Publicações & Melhora infraestrutura da instituição & Transferência de conhecimento \\
\cline { 1 - 2 } & $83 \%$ & $66 \%$ & $41 \%$ \\
U-U & Transf.conhec. & Publicações & Teses/dissertações \\
\hline & $100 \%$ & $91 \%$ & $71 \%$ \\
\hline U-IP & Publicações & Transferência de conhecimento & Contribuições para as comunidades locais \\
\cline { 2 - 3 } & $81 \%$ & $81 \%$ & $54 \%$ \\
\hline
\end{tabular}




\section{CONSIDERAÇÕES FINAIS}

A presente pesquisa buscou caracterizar as cooperações em que a universidade constitui uma das partes envolvidas, mormente, os relacionamentos nos moldes Universidade-Empresa (U-E), Universidade-Universidade (U-U) e Universidade-Instituto de Pesquisa (U-IP). Para tanto, foram avaliados os fatores que interferem nas cooperações com ênfase nos motivadores à cooperação, dificultadores, determinantes da escolha dos parceiros e principais resultados do trabalho cooperativo.

Para os itens que compõem as dimensões de dificultadores, motivadores e determinantes da cooperação interinstitucional, foram individuadas diferenças de acordo com o tipo de parceiro que as instituições acadêmicas se envolvem, constatação que se torna ainda mais evidenciada quando se comparam as relações U-E e U-U. Destacam-se, portanto, os seguintes fatores nas dimensões analisadas:

- motivadores: possibilidade de conquistar apoio financeiro em projetos de pesquisa viabilizando sua execução; possibilidade de melhorar a infraestrutura da instituição no que concerne a aquisição de equipamentos, livros e materiais de laboratório;

- dificultadores: excesso de normas para a comprovação da execução de gastos conforme o acordado no plano de trabalho assinado pelas instituições parceiras; falta de recursos suficientes para a execução dos projetos. Esse último refere-se a recursos humanos, recursos materiais e recursos financeiros;

- determinantes da escolha do parceiro: credibilidade da instituição e/ou do pesquisador representante da instituição, muitas vezes, reconhecido como referência em determinado campo de conhecimento específico; experiências anteriores bem-sucedidas que culminaram no interesse das partes em dar continuidade ao relacionamento frutífero iniciado com base em outras empreitadas.

Observou-se, ainda, que cada tipo de relação possui determinadas especificidades no que se refere ao instrumento contratual que a norteia, predominando - na maioria dos casos - o emprego do convênio ou acordo 
formal de cooperação como principal mecanismo para reger a relação, as obrigações das partes, as contrapartidas e os cronogramas de execução do projeto. Outra constatação concerne, nos casos pesquisados, à predominância de pesquisas conjuntas na área de ciências exatas, independentemente da instituição parceira.

Uma vez identificados os fatores que interferem nas cooperações interinstitucionais, sugere-se para potenciais pesquisas futuras: (I) avaliar o quanto cada fator interfere por intermédio das técnicas de regressão quantílica; (II) examinar os instrumentos contratuais que regem esses relacionamentos. Nesse ponto, as contribuições da teoria dos custos de transação poderiam auxiliar os gestores na construção de instrumentos mais completos; (III) centrar a investigação em instituições de ensino e pesquisa com relações amadurecidas de cooperação com o setor privado, tais como a Pontifícia Universidade Católica do Rio Grande do Sul (sPOLIDORo; AUdY, 2008; FERREIRA; SORIA; CLOSS, 2OI2; NOVELI; SEGATTO, 20I2) OU a Universidade Estadual de Campinas (CESAR, 2009). Por fim, uma vez apontados quais são os resultados das cooperações interinstitucionais considerados mais relevantes para os respondentes pertencentes às instituições acadêmicas, sugere-se (Iv) averiguar quais são as implicações das cooperações sob a ótica das empresas e dos institutos de pesquisa. Como limitações da pesquisa, aponta-se o tamanho da amostra e a concentração da coleta dos dados em apenas um estado brasileiro. 


\section{REFERÊNCIAS}

AGUIAR, A. C. Percepções de pesquisadores sobre atividades cooperativas de P\&D: uma análise com base na teoria institucional. Revista de Administração Contemporânea, v.11, n.4, p.59-86, 2007.

ARZA, V. Channels, benefits and risks of public-private interactions for knowledge transfer: a conceptual framework inspired by Latin America. Science and Public Policy, v.37, n.7, p.473-484, 2010.

BECKER, J. L.; DA JORNADA; J. A. H.; RITTER, F.J. A conceptual framework for understanding the interaction between academic research and industry. In: INTERNATIONAL CONFERENCE ON PHYSICS AND INDUSTRIAL DEVELOPMENT: Bridging The Gap, 2, 1996, Belo Horizonte. Anais... Belo Horizonte: World Scientific, Brazil, 1996.

BONACCORSI, A.; PICCALUGA, A. A theoretical framework for the evaluation of university-industry relationships. R\&D Management, v.2, n.3, p.229-247, 1994. Doi: 10.1111/j.1467-9310.1994.tb00876.x

BOHRER, M.B.A.; ÁVILA, J.; CASTRO, A.C.; CHAMAS, C.I.; CARVALHO, S.M.P. Ensino e pesquisa em propriedade intelectual no Brasil. Revista Brasileira de Inovação, p. 281-310, 2007.

BRASIL. Decreto-Lei 10.973, de 2 de dezembro de 2004. Dispõe sobre incentivos a inovação e à pesquisa científica no ambiente produtivo, e dá outras providências. Brasília, DF: Diário Oficial da República Federativa do Brasil, 2004.

BRASIL. Decreto n ${ }^{\circ}$. 5.563, de 11 de outubro de 2005. Regulamenta a lei 10.973, de 2 de dezembro de 2004, que dispõe sobre incentivos a inovação e à pesquisa científica no ambiente produtivo, e dá outras providências. Brasília, DF: Diário Oficial da República Federativa do Brasil, 2005.

CAPES - COORDENAÇÃO DE APERFEIÇOAMENTO DE PESSOAL DE NÍVEL SUPERIOR. Contribuição da pós-graduação brasileira para o desenvolvimento sustentável Capes na Rio+20. Brasília: CAPES, 2012a.

CAPES - COORDENAÇÃO DE APERFEIÇOAMENTO DE PESSOAL DE NÍVEL SUPERIOR. Edital professor visitante do exterior 2012. Edital CAPES 05/2012. 2012b. Disponível em: < http:// capes.gov.br/images/stories/download/editais/ Edital_005-2012_PVE.pdf >. Acesso em: 02/05/2015.

CESAR, J. Avaliação do potencial de mercado de tecnologias nascentes: a experiência do Programa de Investigação Tecnológica na Unicamp. In: SANTOS, M. E. R.; TOLEDO, P. T. M.; LOTUFO, R. A. (Orgs.). Transferência de tecnologia: estratégias para estruturação e gestão de Núcleos de Inovação Tecnológica. Campinas: Komedi, 2009. p.217-254.

COSTA, P. R.; PORTO, G. S.; FELDHAUS, D. Gestão da cooperação empresauniversidade: o caso de uma multinacional brasileira. Revista de Administração Contemporânea, v.14, n.1, p.100-121, 2010. 
CYERT, R. M.; GOODMAN, P. S. Creating effective university-industry alliances: an organizational learning perspective. Organizational Dynamics, v.25, n.4. p.45-57, 1997.

DOSSA, A.; SEGATTO, A. P. Pesquisas cooperativas entre universidades e institutos públicos no setor agropecuário brasileiro: um estudo na Embrapa. Revista de Administração Pública, v.44, n.6, p. 1327-1352, 2010.

ETZKOWITZ, H.; LEYDESDORFF, L. The dynamics of innovation: from National Systems and "Mode 2" to a Triple Helix of university-industry-government relations. Research Policy. v.29, n.1, p.109-123, 2000.

ETZKOWITZ, H. The Triple Helix of University-Industry-Government: Implications for Policy and Evaluation. Science Policy Institute: SISTER, 2002.

FAULKNER, W.; SENKER, J.; VELHO, L. Knowledge frontiers: public sector research and industrial innovation in biotechnology, engineering ceramics, and parallel computing. New York: Oxford University Press, 1995.

FERNANDES, A. C.; CAMPELLO DE SOUZA, B.; STAMFORD SILVA, A.; SUZIGAN, W.; CHAVES, C.; ALBUQUERQUE, E. Academy-industry links in Brazil: evidence about channels and benefits for firms and researchers. Science and Public Policy, v.37, n.7, p. 485498, 2010.

FERREIRA, G. C.; SORIA, A. F.; CLOSS, L. Gestão da interação Universidade-Empresa: o caso PUCRS. Sociedade e Estado, Brasília , v.27, n.1, p.79-94, 2012 .

FINK, A. The survey handbook. London: Sage Publications, 1995.

FREITAS, I. M. B.; MARQUES, R. A.; SILVA, E. M. P. University-industry collaboration and innovation in emergent and mature industries in new industrialized countries.

Research Policy, v.42, n.2, p.443-453, 2013.

GATTI, B. A. Formação de grupos e redes de intercâmbio em pesquisa educacional: dialogia e qualidade. Revista Brasileira de Educação, n.30, 124-132, 2005. Doi: 10.1590/S141324782005000300010

HAIR, J. F.; ANDERSON, R. E.; TATHAM, R. L.; BLACK, W. C. Análise Multivariada de Dados. 5.ed. Porto Alegre: Bookman, 2005.

LOTUFO, R. A. A institucionalização de Núcleos de Inovação Tecnológica e a experiência da Inova Unicamp. In: SANTOS, M. E. R.; TOLEDO, P. T. M.; LOTUFO, R. A. (Orgs.). Transferência de tecnologia: estratégias para estruturação e gestão de Núcleos de Inovação Tecnológica. Campinas: Komedi, 2009. p.41-73.

LUNDVALL, B. A. National systems of innovations: towards a theory of innovation and interactive learning. London: Pinter Publishers, 1992.

McDANIEL, C.; GATES, R. Pesquisa de Marketing. São Paulo: Pioneira Thomson Learning, 2003. 
MELLO, C. M.; CRUBELLATE, J. M.; ROSSONI, L. Dinâmica de Relacionamento e Prováveis Respostas Estratégicas de Programas Brasileiros de Pós-Graduação em Administração à Avaliação da Capes: Proposições Institucionais a partir da Análise de Redes de Co-Autorias. Revista de Administração Contemporânea, Curitiba, v.14, n.3, art. 3, p. 434-457, 2010.

MEYER-KRAHMER, F.; SCHMOCH, U. Science-based technologies: industry-university interactions in four fields. Research Policy, v.27, n. 8, p.835-851, 1998

MORA-VALENTIN, E.; MONTORO-SANCHEZ, A.; GUERRAS-MARTIN, L.

Determining factors in the success of $R \& D$ cooperative agreements between firms and research organizations. Research Policy, n.33, p.17-40, 2004.

MUSSI, F. B.; SEGATTO, A. P. Análise do instrumento de cooperação interinstitucional Programa de Pesquisadores visitantes em Instituições de Ensino, pesquisa e/ ou extensão. Revista de Administração e Inovação, v.10, n.3, p.115-142, 2013.

NOVELI, M.; SEGATTO, A. P. Processo de cooperação universidade-empresa para a inovação tecnológica em um parque tecnológico: evidências empíricas e proposição de um modelo conceitual. Revista de Administração e Inovação, São Paulo, v.9, n.1, p.81-105, 2012.

PLONSKI, G. A. Cooperação Empresa-Universidade na Íbero-América: Estágio Atual e Perspectivas. Revista de Administração. São Paulo, v.30, n.2, p.65-74, 1995.

PLONSKI, G. A. Cooperação universidade empresa: um desafio gerencial complexo. Revista de Administração, São Paulo, v.34, n.4, p.5-12, 1999.

PORTO, G. S.; PLONSKI, G. A. A informação na decisão empresarial de cooperação com a universidade. In: SIMPÓSIO DE GESTÃO DA INOVAÇÃO TECNOLÓGICA, 21., 2000, São Paulo, Anais... São Paulo, ANPAD, 2000.

PRAGER, O. J.; OMENN, G. S. Research, innovation and university-industry linkages. Science, v.207, n.25, p.379-384, 1980.

RAPINI, M. S.; SUZIGAN, W.; FERNANDES, A. C.; DOMINGUES, E.; CARVALHO, S. S. M.; CHAVES, C. V. A contribuição das universidades e institutos de pesquisa para o Sistema de Inovação Brasileiro. In: ENCONTRO NACIONAL DE ECONOMIA, 37. 2009, Foz do Iguaçu. Anais... Foz do Iguaçu: ANPEC, 2009.

ROSSONI, L.; GUARIDO FILHO, E. R. Cooperação Interinstitucional no Campo da Pesquisa em Estratégia. RAE. Revista de Administração de Empresas, v.47, p.72-86, 2007.

ROSSONI, L.; HOCAYEN-DA-SILVA, A. J.; FERREIRA JUNIOR, I. Estrutura de Relacionamento entre Instituições de Pesquisa do Campo de Ciência e Tecnologia no Brasil. RAE. Revista de Administração de Empresas, v. 48, p. 34-48, 2008.

ROSSONI, L.; HOCAYEN-DA-SILVA, A. J. Cooperação entre Pesquisadores da Área de Administração da Informação: Evidências Estruturais de Fragmentação das Relações no Campo Científico. RAUSP. Revista de Administração, v.43, p.138-151, 2008.

SANTOS, M. E.; SOLLEIRO, J. L.; LAHORGUE, M. A. Boas práticas de gestão em 
escritórios de transferência de tecnologia. In: SIMPÓSIO DE GESTÃO DA INOVAÇÃO TECNOLÓGICA, 23, 2004. Curitiba, Anais...Curitiba, 2004.

SEGATTO, A. P. Análise do processo de cooperação universidade-empresa: um estudo exploratório. 1996. 175fls.. Dissertação (Mestrado em Administração) - Departamento de Administração, Universidade de São Paulo, São Paulo, 1996.

SEGATTO-MENDES, A. P.; SBRAGIA, R. O processo de cooperação universidadeempresa em universidades brasileiras. Revista de Administração, São Paulo, v.37, n.4, p.5871, 2002.

SHIMA, W. T.; SCATOLIN, F. D. Uma comparação das universidades/institutos de pesquisa e das empresas sobre o processo de interação. Revista de Economia, v.37, n. especial, p.213-238, 2011.

SPENDER, J. C. A relação universidade-empresa - desafios e oportunidades na geração e na disseminação do conhecimento. Mesa redonda. Revista de Administração, v.34, n.4, p.32-45, 1999.

SPOLIDORO, R.; AUDY, J. Parque cientifico e tecnológico da PUCRS : TECNOPUC. Porto Alegre: EDIPUCRS, 2008.

WRIGHT, R. How to get the most from universities relationships. Sloan Management Review, v.49, n.3, p.74-81, 2008.

YIN, R. K. Estudo de caso: planejamento e métodos. 3.ed. Porto Alegre: Bookman, 2001. 


\section{DADOS DOS AUTORES}

\section{FABRICIO BARON MUSSI ${ }^{\star}$ fabricio_mussi@hotmail.com}

Doutorando em Administração pela PUC/PR

Instituição de vinculação: Pontifícia Universidade Católica do Paraná

Curitiba/PR - Brasil

Áreas de interesse em pesquisa: Cooperação interinstitucional; sustentabilidade; inovação.

${ }^{\star}$ Rua Imaculada Conceição, 1155-Bloco Acadêmico, Sala 103B, $1^{\circ}$ Andar

Prado Velho Curitiba/PR 80215-901 